\title{
Circulating mitochondria in deceased organ donors are associated with immune activation and early allograft dysfunction
}

\author{
Justin Pollara, ${ }^{1}$ R. Whitney Edwards, ${ }^{1}$ Liwen Lin, ${ }^{1}$ Victoria A. Bendersky, ${ }^{2}$ and Todd V. Brennan ${ }^{3}$ \\ 'Department of Surgery, Duke University Medical Center, Durham, North Carolina, USA. ²Department of Medicine, \\ Duke University School of Medicine, Durham, North Carolina, USA. ${ }^{3}$ Department of Surgery, Cedars-Sinai Medical Center, \\ Los Angeles, California, USA.
}

Brain death that occurs in the setting of deceased organ donation for transplantation is associated with systemic inflammation of unknown origin. It has recently been recognized that mitochondriaderived damage-associated molecular patterns (mtDAMPs) released into the circulation in the setting of trauma and tissue injury are associated with a systemic inflammatory response. We examined the blood of deceased organ donors and found elevated levels of inflammatory cytokines and chemokines that correlated with levels of mtDAMPs. We also found that donor neutrophils are activated and that donor plasma contains a neutrophil-activating factor that is blocked by cyclosporin $\mathrm{H}$, a formyl peptide receptor-1 antagonist. Examination of donor plasma by electron microscopy and flow cytometry revealed that free- and membrane-bound mitochondria are elevated in donor plasma. Interestingly, we demonstrated a correlation between donor plasma mitochondrial DNA levels and early allograft dysfunction in liver transplant recipients, suggesting a role for circulating mtDAMPs in allograft outcomes. Current approaches to prolong allograft survival focus on immune suppression in the transplant recipient; our data indicate that targeting inflammatory factors in deceased donors prior to organ procurement is another potential strategy for improving transplant outcomes.

Conflict of interest: The authors have declared that no conflict of interest exists.

Submitted: April 12, 2018

Accepted: June 21, 2018

Published: August 9, 2018

Reference information: JCI Insight. 2018;3(15):e121622. https://doi.org/10.1172/jci. insight.121622.

\section{Introduction}

Organ transplantation is the definitive treatment for end-stage organ disease. Despite significant advances in our understanding of immune mechanisms and immunosuppression therapy, $25 \%-40 \%$ of grafts will be lost within 5 years of transplantation depending on graft type (1), and immune-mediated graft rejection remains the leading cause of organ loss. While nearly 30,000 organ transplants are performed in the United States each year, the waitlist for transplant organs currently exceeds 123,000 individuals and is rising (1). Given the shortage of available organs, there is a critical need for improving outcomes.

Retrospective studies have demonstrated that living donor transplants have superior outcomes compared with deceased donor transplants in terms of immediate graft function and long-term survival (2-5). It is well-known that donor brain death results in a systemic inflammatory response (5) that can increase graft immunogenicity (6) and is associated with inferior posttransplant outcomes (7). However, there is presently a gap in knowledge regarding the specific cellular and molecular pathways responsible for the initiation of the deceased donor inflammatory response.

Tissue injury releases endogenous damage-associated molecular patterns (DAMPs) that can act as potent activators of the innate immune system $(8,9)$. A diverse array of DAMPs derived from damaged cells and extracellular matrix has been described $(3,10-12)$, and recent research has highlighted mitochondria as a major source of cellular DAMPs (13-20). Mitochondria are the remnant of an endosymbiotic relationship between eukaryotic cells and aerobic Rickettsia-like bacteria that occurred approximately 2 billion years ago $(21,22)$. Due to their bacterial origin, mitochondria retain DAMPs that resemble bacterial pathogen-associated molecular patterns (PAMPs), including N-formyl-methionyl peptides (NFPs) and DNA with unmethylated CpG motifs that activate formyl-peptide receptor 1 (FPR1) and TLR9, respectively $(23,24)$.

There is evidence that extracellular mitochondria contribute to inflammation, immunity, and response to tissue injury $(14,15,18)$. Elevated levels of mitochondrial DNA (mtDNA) have been detected in the plasma 
of patients following trauma $(25,26)$, in patients with myocardial $(27,28)$ and neurologic tissue injury $(29$, 30 ), and in setting of sepsis $(31,32)$. However, the role of mitochondria-derived DAMPs (mtDAMPs) has not been investigated in the setting of brain death, nor has their effect on allograft function been described.

In this study, we examined the inflammatory response in deceased organ donors in terms of polymorphonuclear cell (neutrophil [PMN]) activation and levels of circulating cytokines and chemokines. We found that PMNs are often highly activated in deceased donors and are accompanied by elevated levels of donor cytokines and chemokines that correlate with levels of circulating mtDAMPs. Within donor blood, we identified NFPs as the main PMN-activating mtDAMP and demonstrate that the FPR-1 inhibitor, cyclosporin $\mathrm{H}$ $(\mathrm{CsH})$, blocks PMN activation by donor blood. Finally, we examined deceased donor outcomes following transplantation and show an association between donor circulating mtDNA levels and early allograft dysfunction (EAD) in liver allograft recipients. These findings suggest that mitochondria contribute to the deceased donor inflammatory response and can affect allograft function following transplantation.

\section{Results}

Study cohort. Plasma, serum, and whole blood were collected from 65 deceased organ donors immediately prior to initiation of organ procurement procedures. Within the cohort, 55 donors met donation after brain death (DBD) criteria and 10 donors met donation after cardiac death (DCD) criteria. As shown in Table 1, there were no significant differences between DBD and DCD donors for key study demographics.

Activated PMNs are present in the circulation of deceased organ donors. Because PMNs are critical early mediators of innate immune responses, we first sought to determine if peripheral blood PMNs were activated in deceased donors. We used direct immunofluorescence staining and flow cytometry to define the activation profiles of PMNs present in peripheral blood collected from deceased organ donors immediately prior to initiation of organ procurement procedures. An example of the gating strategy used to identify PMNs is shown in Figure 1A. PMNs were defined as large-granular CD3-, CD19-, CD14- ${ }^{-} \mathrm{CD}^{-} 6 b^{+}$leukocytes. PMNs present in peripheral blood collected from living healthy blood donors $(n=5)$ were used to establish baseline expression levels of cell surface markers of PMN activation. Examples of the downregulation of CD16 and CD62L (L selectin) as well as the upregulation of CD11b and CD66b are shown (Figure 1A). To quantify these differences, we determined the ratios of median fluorescent intensities (MFIs) of each PMN activation marker relative to the MFIs of nonactivated healthy donor PMNs (Figure 1B). By this method, MFI ratios $>1$ indicate higher cell surface expression on PMNs from deceased donors relative to healthy living controls, and ratios $<1$ indicate lower expression. We observed that PMNs from deceased organ donors have a cell surface phenotype consistent with cellular activation when compared with healthy controls, as shown by increased MFI for CD11b (Figure 1C) and CD66b (Figure 1D). PMNs from deceased donors also expressed significantly higher levels of cell surface CD89 and CD35 as well as decreased CD16 and CD62L levels compared with healthy controls (Supplemental Figure 1; supplemental material available online with this article; https://doi.org/10.1172/jci.insight.121622DS1). We observed similar clustering of PMN markers between DBD and DCD donors, suggesting that brain death and cardiac death are similarly able to cause PMN activation.

Plasma from deceased donors can activate PMNs from healthy living donors. We next asked if there were soluble factors present in the plasma of deceased donors that are capable of activating PMNs from healthy living donors ex vivo. To asses this, we coincubated plasma from deceased organ donors with leukocyte preparations from healthy normal donors (NDs) overnight and then evaluated the expression levels of cell surface activation markers as described above. Whole leukocyte preparations were used in favor of purified PMNs in order to minimize PMN manipulation and to more accurately recapitulate in vivo conditions. Incubation of healthy control PMNs with autologous plasma was used as the baseline control for calculations of MFI ratios. Heterologous plasma from healthy NDs were included as negative controls. Supplemental Figure 2 shows the gating strategy used for analysis of PMNs, and the viability of PMNs at time of analysis (median viability $=87 \%$ ). As shown in Figure $2 \mathrm{~A}$, plasma samples from DBD and DCD donors were able to induce upregulation of multiple surface markers of activation, including significant increases in levels of cell surface CD11b (Figure 2B), CD66b, CD16, CD62L, and CD35 (Supplemental Figure 3). The activation profile differed from what was observed for donor PMNs, in that CD89 levels did not increase and that CD16 and CD62L levels increased rather than decreased. This pattern of activation may be related to differences between acute and chronic PMN activation kinetics. To rule out contamination with endotoxin (LPS), a limulus amoebo- 


\section{Table 1. Demographics of study cohort}

\begin{tabular}{|c|c|c|c|c|}
\hline Characteristics $^{A}$ & $\mathrm{DBD}(n=55)$ & $\operatorname{DCD}(n=10)$ & Total $(n=65)$ & $P$ value $^{\mathrm{B}}$ \\
\hline Missing & $1(1.82 \%)$ & $2(20 \%)$ & $3(4.62 \%)$ & \\
\hline Missing & $1(1.8 \%)$ & $2(20.0 \%)$ & $3(4.6 \%)$ & \\
\hline Race & & & & 0.56 \\
\hline White & 39 (70.9\%) & $7(70 \%)$ & 46 (70.8\%) & \\
\hline BMI & $26.4(23.2,33.4)$ & $29.3(24.3,31.8)$ & $26.6(23.2,32.4)$ & 0.91 \\
\hline Missing & $1(1.82 \%)$ & $2(20 \%)$ & $3(4.62 \%)$ & \\
\hline KDPI & $63(26,90)$ & $54(40.5,67.5)$ & $63(28,85)$ & 0.98 \\
\hline Missing & $2(3.64 \%)$ & $2(20 \%)$ & $4(6.15 \%)$ & \\
\hline Cause of death & & & & 0.38 \\
\hline Mechanism of injury & & & & 0.80 \\
\hline Intracranial & $21(38.9 \%)$ & $2(25 \%)$ & $23(37.1 \%)$ & \\
\hline Traumatic & $12(22.2 \%)$ & $2(25 \%)$ & $14(22.6 \%)$ & \\
\hline Other & $21(38.9 \%)$ & $4(50 \%)$ & $25(40.3 \%)$ & \\
\hline Missing & 1 & 2 & 3 & \\
\hline Diabetes & $8(15.1 \%)$ & $1(12.5 \%)$ & $9(14.8 \%)$ & 1.00 \\
\hline Missing & 2 & 2 & 4 & \\
\hline Steroids & $45(86.5 \%)$ & $2(25.0 \%)$ & $47(78.3 \%)$ & 0.0008 \\
\hline Missing & 3 & 2 & 5 & \\
\hline White blood cell count & $12.8(9.7,15.7)$ & $12.3(10,20.7)$ & $12.8(9.7,16.5)$ & 0.71 \\
\hline
\end{tabular}

cyte lysate (LAL) test was performed on the donor plasma samples, and we were able to confirm the absence of endotoxin (Supplemental Figure 4). Overall, the observed changes in the expression of cell surface membranes proteins indicates that deceased donor plasma is able to cause PMN activation.

We next considered whether overall donor quality was associated with the ability of donor plasma to activate healthy donor PMNs. The Kidney Donor Profile Index (KDPI) is a numerical measurement based on the Kidney Donor Risk Index that combines 10 donor factors, including clinical parameters and demographics, to summarize the quality of deceased donor kidneys relative to other recovered kidneys into a single number (33). Thus, it provides a quantitative measurement of donor quality. Using cell surface expression of $\mathrm{CD} 11 \mathrm{~b}$ as a marker of PMN activation, we observed a moderate but significant correlation between KDPI and activation of PMNs by donor plasma (Figure 2C). These data indicate that a donor's health status may contribute to their inflammatory state.

Plasma from deceased organ donors induces ROS production by PMNs. Upon activation, PMNs typically undergo an oxidative burst that results in the production of ROS. We hypothesized that soluble factors present in deceased donor plasma can activate PMNs to produce increased levels of ROS. To test this, we compared ROS production induced by healthy donor PMNs treated ex vivo with plasma from DBD and DCD donors to ROS production induced by healthy donor heterologous and autologous plasma (PMNs and serum from same healthy donor) using a luminescence-based assay. These measurements were compared with baseline and N-formylmethionyl-leucyl-phenylalanine-induced (fMLP-induced) ROS production. A random subset of samples $(n=23 \mathrm{DBD} / \mathrm{DCD}, n=5 \mathrm{ND})$ from our study cohort were 
A
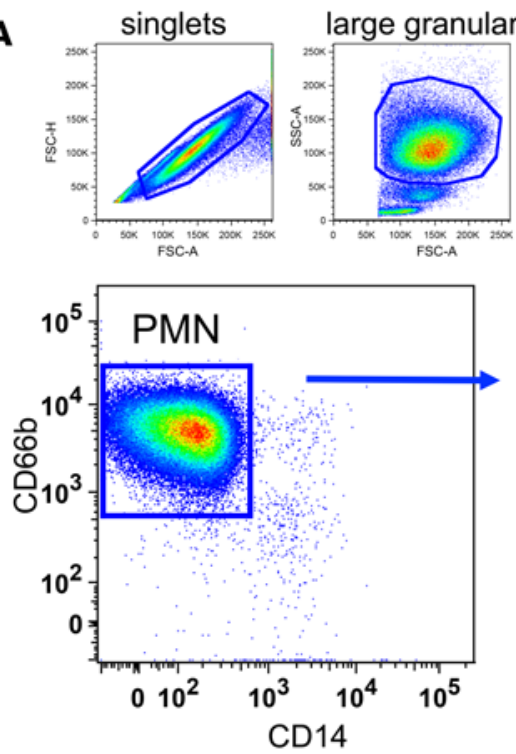

B

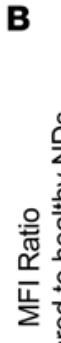

要

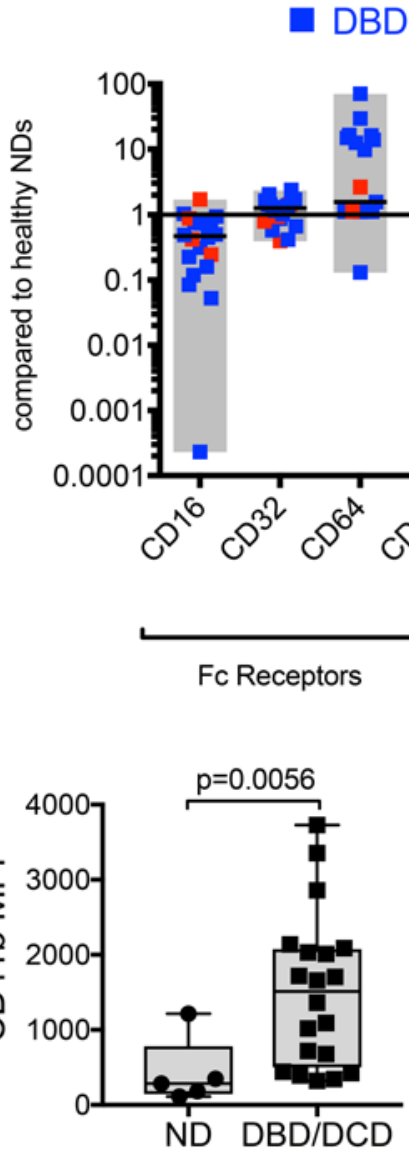

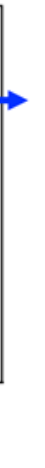
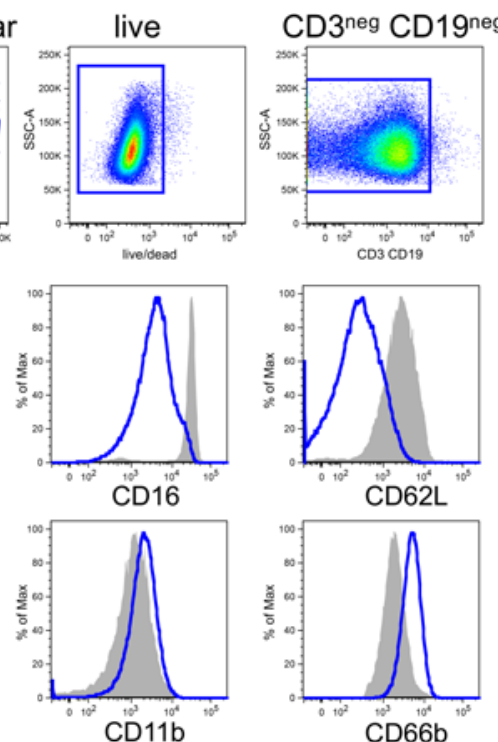

DCD
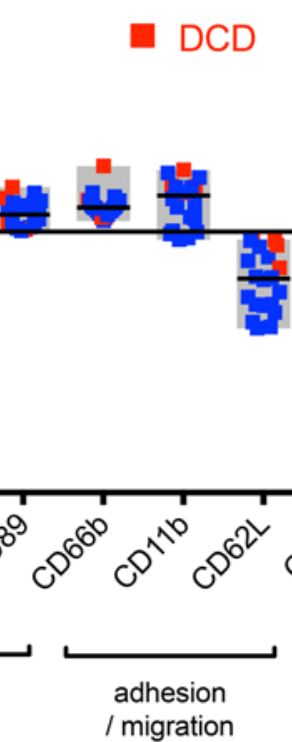

\section{D}

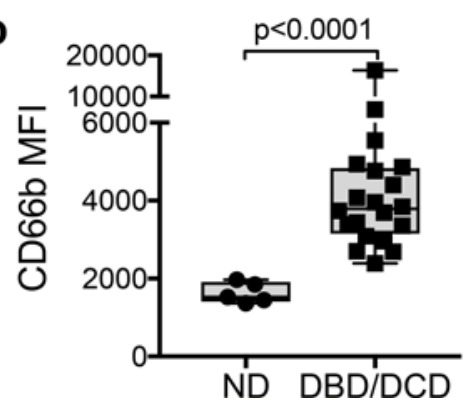

Figure 1. Activated PMNs are present in the circulation of deceased organ donors. (A) Gating strategy used for analysis of PMNs present in peripheral blood collected from deceased organ donors and healthy living normal donors (NDs) after whole blood staining. Histograms show example comparisons of median fluorescence intensity (MFI) for selected activation markers on the surface of deceased donor PMNs (blue lines) and PMNs from a healthy living NDs (gray filled histograms). (B) Cell surface expression levels of the indicated proteins on PMNs from deceased organ donors (donation after brain death [DBD], $n=16$, blue squares; donation after cardiac death [DCD], $n=4$, red squares) were compared with PMNs from healthy living NDs $(n=5)$ using median MFI ratios as described in the Methods. The gray shaded boxes represent the range of observed responses, and the medians are indicated by black lines. MFIs of (C) CD11b and (D) CD66b were significantly higher on $\mathrm{PMN}$ in the circulation of whole blood from DBD/ DCD donors $(n=20)$ when compared with blood from NDs $(n=5)$. In C and $\mathbf{D}$, the gray boxes represent the interquartile range, the lines represent the median, and whiskers indicate the range of observed responses. Comparisons were performed using a Mann-Whitney test. $P<0.05$ was considered significant.

assayed. Examples of the ROS production curves obtained with this assay are shown in Figure 3A. Low basal levels of ROS are produced when PMNs from NDs are incubated with autologous plasma (Figure $3 \mathrm{~A}$, black line) or heterologous plasma from another ND (Figure 3A, blue line). In contrast, plasma from a deceased donor induced a potent ROS response (Figure 3A, red line) that is augmented in response to fMLP stimulation. Interestingly, although all PMNs from healthy NDs produced a robust ROS response to fMLP stimulation, not all donor plasma was found to be activating. Plasma from a different deceased donor (Figure 3A, purple line) was markedly less inflammatory. We selected two points along the kinetic 
A
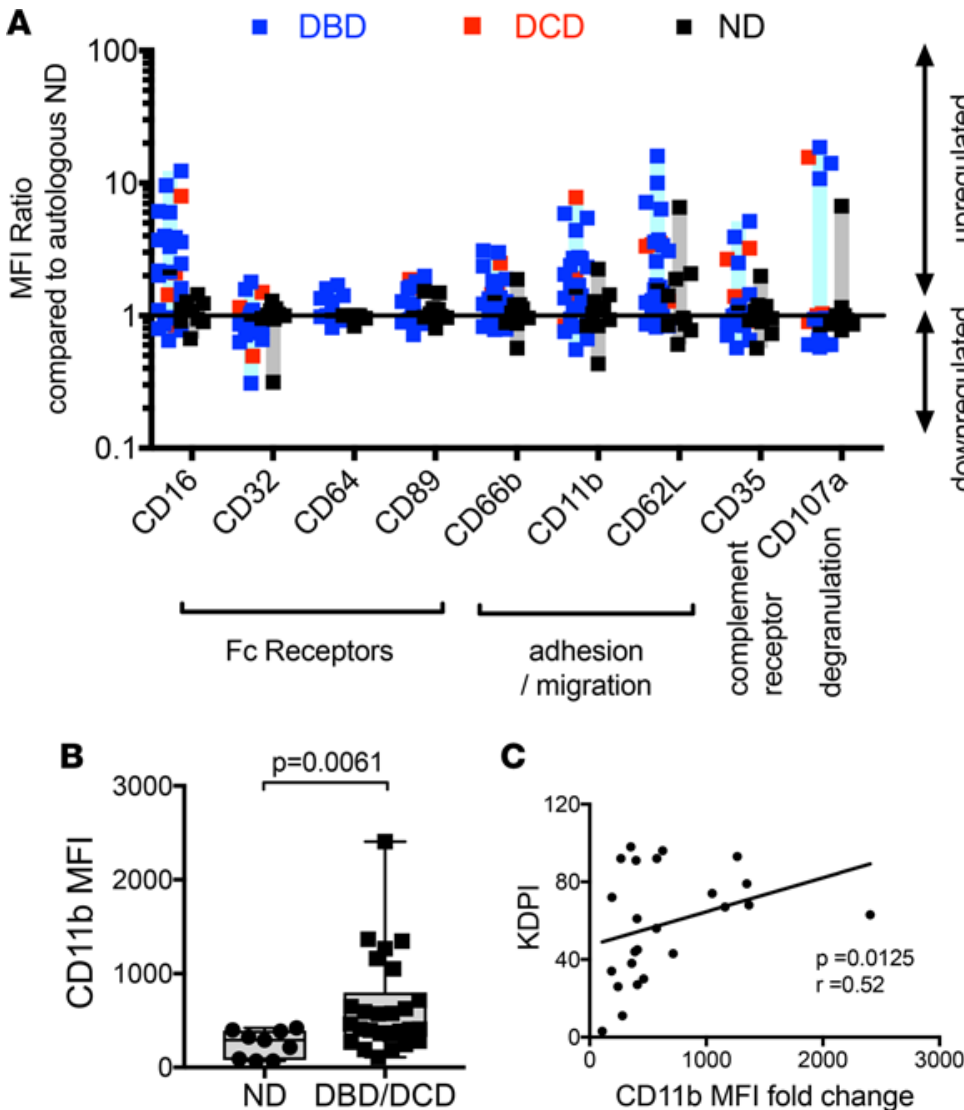

\section{C}

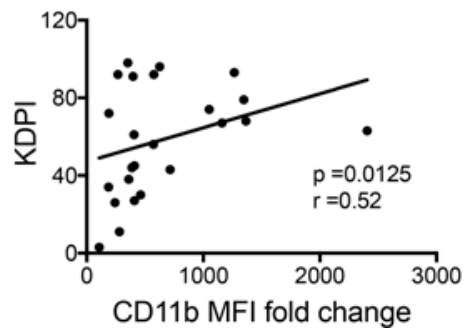

Figure 2. Plasma from deceased organ donors can activate PMNs from healthy living normal donors ex vivo. (A) PMNs from a minimum of 2 healthy living donors were incubated overnight with plasma from deceased organ donors (donation after brain death [DBD], $n=22$, blue squares; donation after cardiac death [DCD], $n=4$, red squares) or healthy normal donors (NDs, $n=9$, black squares), and the average median fluorescence intensity (MFI) ratios compared with incubation with autologous plasma are shown. Shaded boxes represent the range of observed responses and the medians are indicated by black lines. (B) MFIs of CD11b were significantly higher on PMNs incubated with plasma from DBD/DCD donors $(n=26)$ when compared with PMNs incubated with plasma from NDs $(n=9)$. The gray boxes represent the interquartile range, the lines represent the median, and whiskers indicate the range of observed responses. Comparison was performed using a Mann-Whitney test. $P<0.05$ was considered significant. (C) Increased donor kidney donor profile index (KDPI), indicative of poorer donor health, correlates with the ability of donor plasma to activate healthy donor PMNs ex vivo, as assessed by cell surface CD11b expression determined by flow cytometry analysis ( $n=24, \mathrm{KDPI}$ not available for 2 donors). Spearman's rank correlation test was used to examine for correlations between the variables.

ROS curve to facilitate comparisons across samples. These are the "plasma maximum" response and the after fMLP stimulation "new set point" response indicated in Figure 3A. The plasma maximum represents the ability of plasma to induce ROS production, while the new set point provides an indication of ability to promote a persistent ROS response after fMLP stimulation. No significant differences in ROS induction were observed for DBD/DCD donor plasma samples compared with ND plasma samples (Figure 3, A and B; Mann-Whitney test, $P>0.05$ ). However, there was a clear bimodal distribution of the data, and we found that 6 of 23 plasma samples from deceased donors were able to induce ROS production from healthy PMNs, as indicated by plasma maximum responses (Figure 3B, open symbols), while 12 of 23 plasma samples promoted a persistent ROS response after fMLP stimulation, as indicated by new set point responses (Figure 3C, open symbols). Experiments performed using PMNs purified from healthy donor blood incubated with 2 inflammatory donor plasma samples confirmed that ROS production can be induced by direct stimulation of PMNs after depletion other leukocyte populations (Supplemental Figure 5). Collectively, these data indicate that soluble factors present in the circulation of a subset of deceased donors stimulate and potentiate ROS production by PMNs.

Elevated levels of inflammatory molecules in deceased donor serum. We next sought to determine the level of inflammatory molecules in donor sera. We used multiplex assays to profile the cytokine and chemokine levels in sera collected from our study cohort. Data from all 25 cytokines are presented in Supplemental Figure 6. Compared with healthy control plasma, DBD and DCD donor plasma contained significantly higher levels of IL- 6 , IL-8, IL-2 receptor (IL-2R), and IL-1 receptor antagonist (IL-1RA) (Figure 4, A-D) compared with that of ND plasma. Sera from DCD donors also contained significantly higher levels of MIP-1 $\beta$ when compared with NDs (Figure 4E). Levels of IFN- $\alpha$ were similar between NDs and deceased donors (Figure 4F). Overall, these results demonstrate increased levels of inflammation-associated cytokines in the circulation of deceased organ donors.

Mitochondria and mtDAMPs are present in the circulation of deceased organ donors. We hypothesized that mitochondria released from tissue injury and cell death in the setting of deceased organ donation are a major source of DAMPs in the circulation of deceased organ donors. Initially, we measured the amount of NFPs present in sera by ELISA and found that NFPs levels were significantly elevated in sera from deceased donors compared with ND sera (Figure 5). 
A
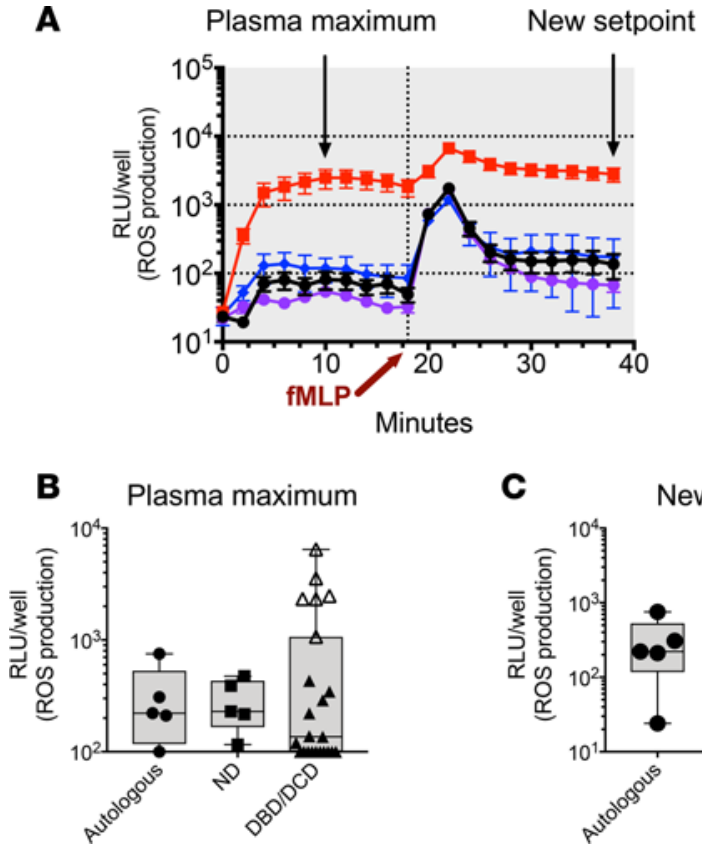

C

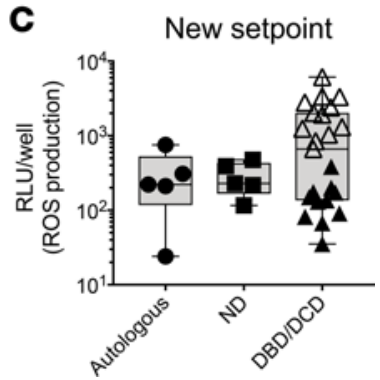

Figure 3. Plasma from deceased organ donors is able to induce production of ROS by PMNs from healthy living normal donors. (A) Example curve of PMN ROS production. Leukocytes from 2 heathy normal donors (NDs) were stimulated with ND plasma (autologous, black line; heterologous, blue line) or plasma from deceased donors (red and purple lines), and ROS production was measured by chemiluminescent detection. Stimulation with $\mathrm{N}$-formylmethionyl-leucyl-phenylalanine (fMLP) is indicated. Data represent the mean and standard error of RLU resulting from oxidation of luminol by ROS. Data points representing the maximum response observed for plasma stimulation and the new set point after plasma and fMLP stimulation along the kinetic curve are indicated. (B) Plasma maximum and (C) new set point ROS production observed for leukocytes from healthy NDs incubated with autologous plasma samples $(n=5)$, plasma from heterologous NDs $(n=5)$, or plasma from deceased donors ( $D B D / D C D, n=23$ ). In $\mathbf{B}$ and $\mathbf{C}$, the gray boxes represent the interquartile range, the lines represent the median, and whiskers indicate the range of observed responses. No significant difference was observed for comparisons between ND and DBD/DCD (Mann-Whitney test). Open symbols in $\mathbf{B}$ and $\mathbf{C}$ represent samples that induced ROS maximum or set point responses above levels observed for NDs.

Next, we used real-time quantitative PCR (RT-qPCR) to measure the amount of mtDNA and genomic DNA (gDNA) present in our donor plasma samples. Concentrations were determined using standard curves derived from purified mtDNA and gDNA of known concentrations. Compared with ND plasma, DBD and DCD donor plasma contained higher levels of both mtDNA and gDNA (Figure 6, A and B). Since mtDNA can serve as an innate immune activator, we next sought to demonstrate a correlation between levels of circulating mtDNA and inflammatory cytokines, as measured by the Luminex assay (Figure 4 and Supplemental Figure 6). As shown in Table 2, mtDNA levels correlated significantly with circulating levels of inflammation-associated cytokines, including IL-6, IL-8, IL-2R, and IL-1Ra. Interestingly, mtDNA levels were not associated with NFP levels or with traumatic brain injury as the mechanism of death (Supplemental Figures 7 and 8).

We next investigated whether DNA detected in plasma was free DNA or complexed within subcellular particles. Centrifugation of the blood for 15 minutes at $1,200 \mathrm{~g}$ during plasma purification excluded intact circulating cells. Further centrifugation of the samples at $12,000 \mathrm{~g}$ for 15 minutes was used to pellet subcellular particles. DNA levels of the resulting supernatant were then reevaluated by RT-qPCR. Interestingly, the majority of the mtDNA was depleted by the higher centrifugation (Figure 6C), while gDNA levels remained unchanged (Figure $6 \mathrm{C}$ ), indicating that the majority of plasma mtDNA was contained within subcellular particles.

As the centrifugal force that pelleted the mtDNA was sufficient to pellet intact whole mitochondria, we next tested for extracellular mitochondria in donor plasma. To do this, we performed flow cytometry analysis on donor plasma stained with antibodies specific for the mitochondrial outer membrane protein, TOMM22, and we examined pelleted subcellular debris by electron microscopy. DBD and DCD donor plasma contained higher levels of TOMM22+ subcellular particles per ml of plasma compared with ND plasma (Figure 6D), and whole intact mitochondria were readily identified by electron microscopy imaging of subcellular debris pelleted from deceased donor plasma (Figure 6E). These data indicate that plasma mtDAMPs may be derived from intact extracellular mitochondria in the circulation.

NFPs present in deceased donor plasma activate PMNs. Healthy donor PMNs were incubated with a subset ( $n=6$, Figure 3B) of deceased donor plasma samples previously shown to induce ROS responses. Pretreatment with 1 and $10 \mu \mathrm{M} \mathrm{CsH}$, an antagonist of FPR-1 (34), resulted in a $20 \%$ and $60 \%$ median decrease in ROS release, respectively (Figure 7). Minimal inhibition was observed after dose reduction of $\mathrm{CsH}$, and control experiments using PMA- and LPS-stimulated PMNs confirmed that inhibition of ROS release by CsH was specific for NFPs (Supplemental Figure 9). Stimulation of PMNs from healthy donors with fMLP resulted in a cell surface phenotype indicative of activation (Supplemental Figure 10) and consistent with the phenotype we observed for PMNs incubated with plasma from deceased organ donors (Figure 2). These data indicate that circulating NFPs can contribute to PMN activation in deceased organ donors. 

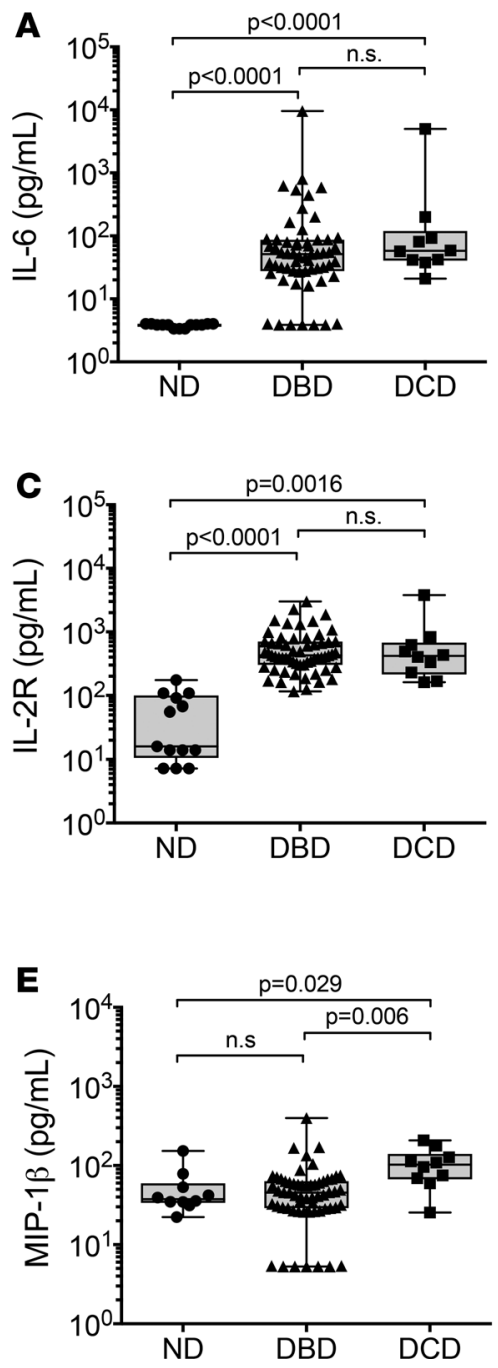
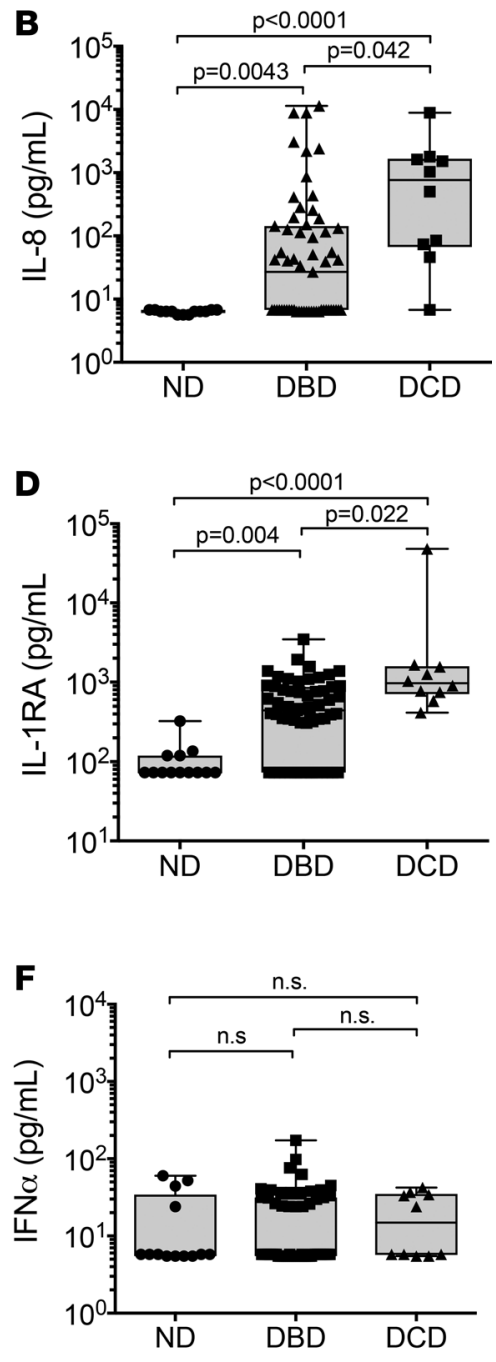

Figure 4. Deceased donors have increased levels of circulating inflammation-associated cytokines. Cytokine levels in the sera of healthy normal donors (NDs, $n=10)$ and deceased donors (donation after brain death [DBD], $n$ = 10; donation after cardiac death [DCD], $n$ $=55$ ) were evaluated by multiplex cytokine assay. (A) IL-6, (B) IL-8, (C) IL-2R, (D) IL-1RA, (E) MIP-1 $\beta$, (F) IFN- $\alpha$. Each data point represents the mean of duplicate assays. The gray boxes represent the interquartile range, the lines represent the median, and whiskers indicate the range of observed responses. Comparisons were performed using a Kruskal-Wallis with Dunn's multiple comparison test. $P<0.05$ was considered significant.

Elevated levels of donor plasma mtDNA are associated with EAD in liver transplant recipients. Mitochondria and mtDAMPs present in the circulation of deceased organ donors may cause allograft inflammation and affect posttransplant allograft function. We next tested whether there was an association between allograft function and donor mtDAMPs, including mtDNA and NFP. Following liver transplantation, EAD was defined as serum aspartate aminotransferase (AST) levels above 1,500 IU/1 within the first 72 hours after transplantation (35). Following kidney transplantation, EAD was defined by the presence of either slow graft function, defined as recipient serum creatinine ( $\mathrm{sCr}$ ) $\geq 3 \mathrm{mg} / \mathrm{dl}$ on postoperative day (POD) 5 , or delayed graft function, defined as the need for dialysis within 7 days of transplantation (36). We found that EAD following liver transplantation was associated with higher levels of donor plasma mtDNA (EAD mean $=10.3 \mathrm{ng} \mathrm{mtDNA} / \mathrm{ml}$, no EAD mean $=5.8 \mathrm{ng} \mathrm{mtDNA} / \mathrm{ml}, P=0.018 ;$ Figure $8 \mathrm{~A})$. In contrast, we found no correlation between donor plasma mtDNA levels and EAD in kidney transplant recipients (Figure $8 \mathrm{~B}$ ). No significant correlation was found between NFP levels and either liver or kidney EAD (Figure 8, C and D) or with serum sCr levels on POD5 following kidney transplantation (Supplemental Figure 11).

\section{Discussion}

The persistent shortage of available organs mandates focused efforts toward identifying strategies to maximize the longevity of each transplant. The majority of donor organs are procured from deceased donors, and these donor organs are more prone to graft dysfunction and rejection when compared with organs from living donors (2-5, 37-39). The underlying reasons for this disparity remain incompletely defined. While variances in overall donor quality can account for a portion of the observed differences in outcomes between living and deceased donor organs, there is also direct evidence that the process of donor brain death plays a role. For 
Table 2. Correlations of plasma NFPs and mtDNA with plasma cytokines/chemokines

\begin{tabular}{|c|c|c|c|c|}
\hline \multirow[t]{2}{*}{ Biomarkers } & \multicolumn{2}{|c|}{ NFPs } & \multicolumn{2}{|c|}{ mtDNA } \\
\hline & Correlation $^{\mathrm{A}}$ & $P$ value $^{B}$ & Correlation $^{A}$ & $P$ value $^{B}$ \\
\hline IL-6 & -0.04 & 0.74 & 0.38 & 0.0009 \\
\hline IL-8 & -0.09 & 0.45 & 0.39 & 0.0005 \\
\hline IL-1b & -0.08 & 0.49 & -0.07 & 0.57 \\
\hline IL-10 & -0.24 & 0.04 & 0.21 & 0.07 \\
\hline IL-13 & -0.09 & 0.42 & -0.09 & 0.44 \\
\hline IL-12 & -0.16 & 0.18 & 0.15 & 0.21 \\
\hline RANTES & -0.13 & 0.26 & 0.10 & 0.41 \\
\hline Eotaxin & -0.14 & 0.24 & 0.10 & 0.37 \\
\hline IL-17 & -0.09 & 0.45 & 0.04 & 0.74 \\
\hline MIP-1 $\alpha$ & -0.12 & 0.32 & 0.12 & 0.32 \\
\hline GM-CSF & -0.06 & 0.64 & -0.09 & 0.46 \\
\hline MIP-1 $\beta$ & -0.17 & 0.14 & 0.25 & 0.03 \\
\hline MCP-1 & -0.17 & 0.16 & 0.12 & 0.32 \\
\hline IL-15 & -0.07 & 0.55 & 0.05 & 0.65 \\
\hline IL-5 & -0.09 & 0.42 & -0.03 & 0.81 \\
\hline IFN- $\gamma$ & -0.02 & 0.87 & 0.44 & $<0.0001$ \\
\hline IFN- $\alpha$ & -0.10 & 0.40 & 0.28 & 0.02 \\
\hline IL-1RA & -0.18 & 0.12 & 0.54 & $<0.0001$ \\
\hline TNF- $\alpha$ & -0.05 & 0.68 & -0.03 & 0.78 \\
\hline IL-2 & 0.04 & 0.76 & -0.09 & 0.45 \\
\hline IL-7 & 0.08 & 0.49 & 0.01 & 0.94 \\
\hline IP-10 & 0.03 & 0.78 & -0.03 & 0.80 \\
\hline IL-2R & 0.18 & 0.13 & 0.27 & 0.02 \\
\hline MIG & -0.08 & 0.51 & 0.29 & 0.01 \\
\hline $\begin{array}{l}\text { IL-4 } \\
\text { ASpearman co } \\
\text { multiple com }\end{array}$ & $\begin{array}{l}-0.07 \\
\text { mines whethe } \\
\text { ted. NFPs, N-f }\end{array}$ & $\begin{array}{l}0.58 \\
\text { is signific }\end{array}$ & $\begin{array}{l}\quad-0.06 \\
\text { ifferent from } 0 . \\
\text { mitochondrial }\end{array}$ & $\begin{array}{l}0.62 \\
\text { ustment for }\end{array}$ \\
\hline
\end{tabular}

example, in a rat kidney transplant model, grafts reject more rapidly when they are procured from rats that have been made brain dead for a period of time, compared with those procured from living donors (38). The ability to closely match donor and recipient factors in these animal experiments, as well as the inclusion of a ventilated non-brain-dead control group, provide strong evidence that brain death is an independent risk factor for allograft rejection.

Consistent with previous studies $(5,40-42)$, we found elevated levels of several cytokines/chemokines, including IL-6, IL-8, soluble IL-2R, soluble IL-1RA, and MIP-1 $\beta$, in the circulation of deceased donors compared with healthy living donors. We also found that deceased donor PMNs had an activated phenotype and that deceased donor plasma had the ability to activate PMNs isolated from healthy blood donors. Our ex vivo experiments were performed with whole leukocyte preparations to minimize PMN manipulation and to more accurately recapitulate the cellular environment of whole blood. It is therefore possible that PMNs could be activated indirectly by signals produced by other leukocytes activated by soluble donor factors. However, our studies with enriched PMN samples that showed similar findings as whole blood argue against a requirement for indirect activation by other leukocytes. Brain death has also been associated with PMN activation in animal models, causing increased PMN infiltrates in liver and kidney allografts, accompanied by increased expression of adhesion molecules $(38,43)$. Additional work using a combination of human samples and animal models will be required to characterize the role of other immune cells in brain death-induced inflammation and PMN activation and to define the mechanism by which activated PMNs may increase allograft immunogenicity.

Our findings support brain death as a systemic inflammatory process, and we sought to determine which factors were responsible for causing this response. Tissue trauma and cell death are common in the setting of deceased organs donors, resulting from traumatic injury (44), and/or the process of brain death itself. Cell death results in the release of DAMPs into the circulation that are able to trigger sterile inflam- 


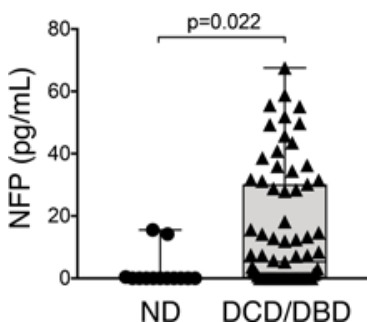

Figure 5. Elevated levels of $\mathbf{N}$-formylated peptides are present in the circulation of deceased organ donors. N-formylated peptides (NFPs) present in sera of healthy normal donors (NDs, $n=10$ ), donation after brain death (DBD) donors $(n=54)$, and donation after cardiac death (DCD) donors $(n=10)$ were measured by ELISA. Each data point represents the mean of duplicate assays. The gray boxes represent the interquartile range, the lines represent the median, and whiskers indicate the range of observed responses. Comparisons were performed using a Mann-Whitney test. $P$ $<0.05$ was considered significant.

matory responses $(9,12,45)$. For example, endogenously derived NFPs are released in the setting of traumatic cell death and cause PMN infiltration into injured tissues $(14,15,18,46,47)$. We found that blockade of the FPR-1 with CsH dramatically reduced the ability of donor plasma to activate healthy control PMNs, inferring that NFPs may be a key innate immune activator in the setting of brain death.

In the absence of bacterial infection, the only endogenous source of plasma NFPs would be mitochondria released from damaged tissue. Mitochondria are a major source of DAMPs (mtDAMPs) and are increasingly recognized as potent innate immune activators $(13,16-18,20,48,49)$. As a result of their origin as bacterial endosymbionts, mitochondria retain prokaryotic molecules that resemble PAMPs. For example, mitochondria contain NFPs and unmethylated DNA CpG motifs, which are ligands for FPR-1 and TLR9, respectively, and which play critical roles in PMN activation and migration to sites of injury $(19,26,47,50,51)$. In addition, mitochondria are the main cellular producers of several NLRP3 inflammasome activators, such as ATP, ROS, and cardiolipin $(21,22,52,53)$, that can lead to IL-1 $\beta$ production, another PMN activator (54).

Considering the multiple PMN-activating properties of mitochondria, we investigated for the presence of extracellular circulating mtDAMPs in deceased donors and discovered elevated levels of mtDNA and NFPs. Interestingly, we found that mtDNA levels correlated with inflammatory cytokines in deceased donors and that this was associated with EAD following liver transplantation. In contrast, we found no association of mtDNA with EAD in kidney transplant recipients, suggesting that kidney allografts may be less effected by circulating mtDAMPs than liver allografts. Further, we detected no significant difference between NFP levels with EAD in liver or kidney transplant recipients. Our observations of elevated levels of mtDNA, NFPs, and intact mitochondria in deceased donor plasma suggests that mtDAMPs contribute to the sterile inflammatory response to brain death, and we therefore expected mtDNA and NFP levels to correlate. However, the detection of NFP is done by an ELISA developed against nonmitochondrial NFP and is less sensitive than the RT-qPCR used for the detection mtDNA. Additional experiments using mass spectrometry-based methods for detecting NFPs may better detect a correlation of NFPs with mtDNA and EAD.

It is important to note that we observed broad variation in the degrees of PMN activation, cytokine levels, and mtDAMP abundance in blood collected from deceased organ donors. This is likely reflective of the diverse heterogeneity in age, health status, cause of death, and other donor factors among our study cohort. Although traumatic injury has been shown to promote the release of DAMPs and to effect organ quality in previous studies $(25,26,55)$, we found no associations between traumatic brain injury and levels of mtDNA or EAD in our cohort. However, we did identify a significant correlation between KDPI and the PMN-activating qualities of donor plasma, indicating that a donor's health status can affect their inflammatory state.

In many cases, organs procured from deceased donors have excellent long-term outcomes similar to those obtained for living donor donors. Consistent with this, we found that not all donor plasma samples contained high levels of mtDAMPs or cytokines, providing evidence that high levels of donor inflammation are not a universal outcome of brain death. With accumulation of additional samples, future work may identify novel correlations among specific donor factors, levels of innate immune activation, and additional DAMPs in deceased organ donors that will better predict the risk of allograft dysfunction and rejection.

Elevated levels of circulating mtDNA in the setting of trauma and neurological injury have been associated with poor clinical outcomes $(19,29,56-60)$. While there is accumulating evidence that circulating mtDNA is associated with systemic inflammation, it has not been previously examined whether mtDNA is circulating as free DNA complexed within subcellular particles, or as free extracellular mitochondria. When we subjected donor plasma to centrifugal forces sufficient to pellet mitochondria, we found that the majority of circulating mtDNA pelleted. This is in comparison to gDNA, which did not. Examination of the pellet by electron microscopy demonstrated that both free mitochondria and mitochondria contained within subcellular vesicles were 
A

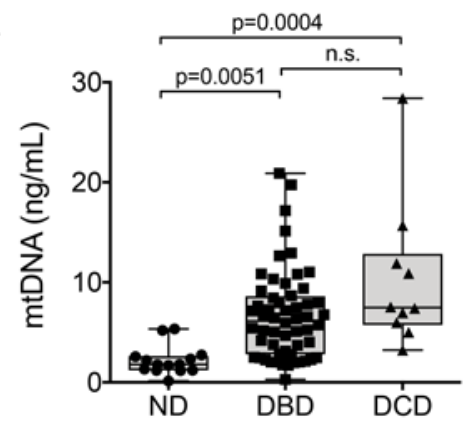

C
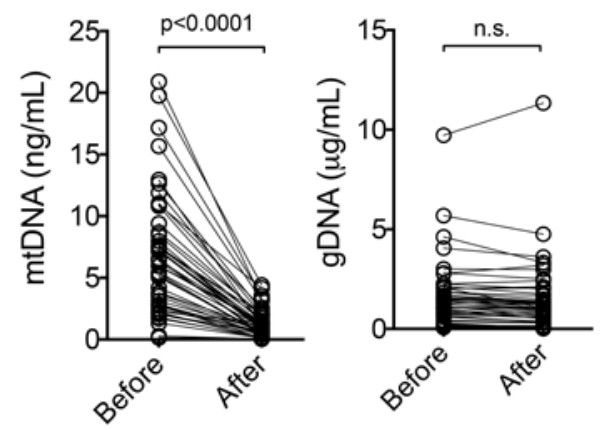

B

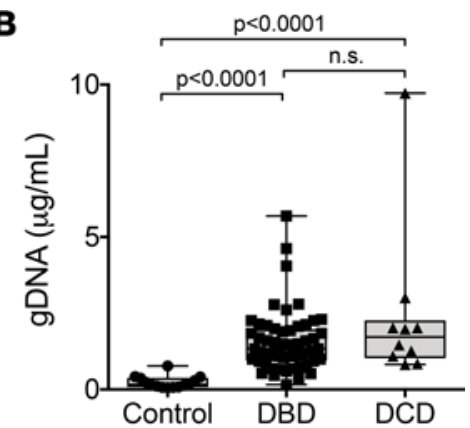

\section{D}

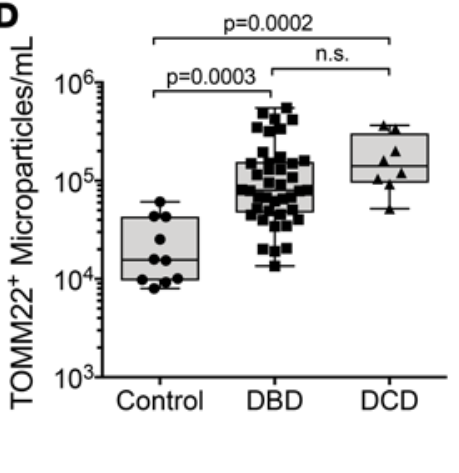

$\mathbf{E}$

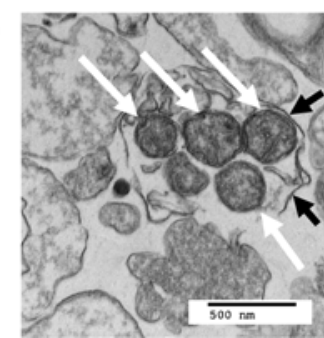

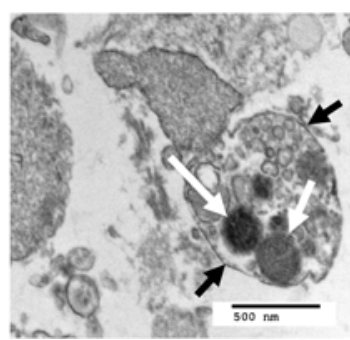

Figure 6. Elevated levels of mitochondrial DNA and whole mitochondria are present in the circulation of deceased organ donors. RT-qPCR was used to measure the amount of (A) mitochondrial DNA (mtDNA) and (B) genomic DNA (gDNA) present in plasma from healthy normal donors (NDs, $n=10$ ) and deceased donors (donation after brain death [DBD], $n=55$; donation after cardiac death [DCD], $n=10$ ). (C) The majority of mtDNA, but not gDNA, was depleted from donor plasma $(n=48$ ) by centrifugation. (D) Plasma from deceased donors ( $D B D, n=41$; DCD, $n=8$ ) contain higher levels of microparticles per $\mathrm{ml}$ of plasma positive for TOMM22 compared with plasma from healthy NDs $(n=10)$. (E) Electron microscopy confirmed the presence of whole intact mitochondria (white arrows) in serum samples collected from deceased organ donors. Mitochondria were observed either within membrane vesicles (black arrows in left and center panels) or free (right panel). Scale bar: $500 \mathrm{~nm}$. In A, B, and D gray boxes represent the interquartile range, the lines represent the median, and whiskers indicate the range of observed responses. Comparisons were performed using a Kruskal-Wallis with Dunn's multiple comparison test (A, B, and $\mathbf{D})$ or Wilcoxon matched-pairs signed-rank test (C). P $<0.05$ was considered significant.

present. Furthermore, flow cytometry analysis of pelleted donor plasma clearly demonstrated microparticles that were positive for TOMM22, a mitochondrial outer membrane protein. Similar to mtDNA, TOMM22+ particles were significantly elevated in deceased donors compared with healthy controls. These data suggest that circulating extracellular mitochondria may be a significant source of circulating mtDNA.

The identification of specific DAMPs responsible for inflammation in deceased donors may allow for the development of specific interventions that reduce graft immunogenicity and improve graft function and longevity. While our study demonstrates a correlation between mtDNA and donor inflammation, it does not establish that circulating mtDAMPs directly cause donor inflammation or are just a marker of tissue injury or inflammation. Similarly, we cannot conclude from our study whether circulating mitochondria directly cause EAD or may merely serve as a biomarker for the development EAD. Studies involving the blockade of donor mtDAMPs, or animal transplant studies involving the pretreatment of donors with mtDAMPs, will better define the role of mitochondria in donor inflammation and posttransplant allograft function. 


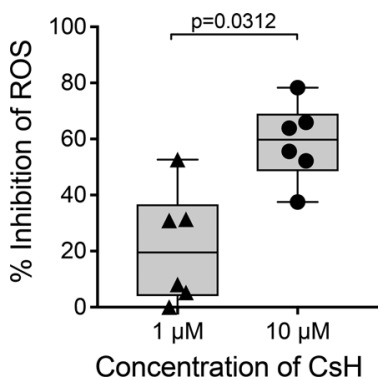

In summary, we have identified the presence of mtDAMPs in the circulation of deceased organ donors and have demonstrated an association between circulating mtDNA and the inflammatory response to brain death as well as allograft function following liver transplantation. We have further identified NFPs as a specific donor mtDAMP that activates PMNs. Further investigation will determine if other donor DAMPs, such as HMGB1 (55), also correlate with donor inflammation and whether detecting a combination of DAMPs is more highly predictive of graft outcomes.

\section{Methods}

Sample collection and processing. Peripheral blood was collected from healthy donors and deceased donors by venipuncture. Peripheral blood samples were collected from deceased organ donors in the operating room prior to organ procurement. Acid citrate dextrose (ACD) tubes were collected for preservation of plasma and peripheral blood leukocytes, and serum separator tubes were used to collect sera (BD). Blood was stored at $4^{\circ} \mathrm{C}$ for less than 24 hours prior to processing. Plasma and sera were isolated by centrifugation for 10 minutes at $500 \mathrm{~g}$ to separate cells, followed by transfer to a $15-\mathrm{ml}$ conical tube and subsequent centrifugation at 1,200 $\mathrm{g}$ for 15 minutes to deplete platelets. Plasma and serum samples were aliquoted and stored at $-80^{\circ} \mathrm{C}$ until use. The remaining cell fraction in the ACD collection tube served as the source of fresh peripheral blood leukocytes used in the assays described below.

Immunoprofiling of peripheral PMNs by whole blood flow cytometry. The phenotype and activation profile of PMNs present in the plasma-depleted cell fraction of peripheral blood of deceased organ donors and healthy living NDs were determined by flow cytometry after staining with the panel of fluorescently conjugated monoclonal antibodies described in Supplemental Table 1. For staining, $100 \mu 1$ plasma-depleted blood was incubated with $100 \mu$ monoclonal antibodies at room temperature for 25 minutes. Next, red blood cells were lysed by incubation with BD Pharm Lyse (BD Biosciences) for 15 minutes at room temperature. The cells were then washed with $1 \%$ FBS in PBS and stained with the Aqua LIVE/DEAD Fixable Aqua Dead Cell Stain Kit (dilution 1:800, Thermo Fisher Scientific) for 20 minutes to permit identification of viable cells. The cells were washed again with $1 \%$ FBS PBS, fixed in $1 \%$ paraformaldehyde PBS, and acquired using a BD Fortessa flow cytometer (BD Biosciences) running FACSDiva acquisition software (BD Biosciences). The cytometer was rigorously maintained with quality control procedures regularly performed as described by Perfetto and colleagues (61). Flow cytometry data analysis was performed using FlowJo 9.9.4 software. Gates were established using fluorescence minus one staining mixture. To compare expression levels on cells isolated from deceased donors to those isolated from healthy living NDs, we calculated the ratio of the MFIs of each interrogated protein on the cell surface of PMNs present in the blood of deceased donors with respect to the mean of MFIs observed for PMNs from healthy donors.

Ex vivo bioassay for activation of healthy donor PMNs by deceased donor plasma. Red blood cells were lysed from plasma-depleted ACD-preserved blood of healthy living NDs using BD Pharm Lyse according to manufacturer's recommended protocol (BD Biosciences). $100 \mu \mathrm{l}$ of the leukocytes and $100 \mu \mathrm{l}$ of plasma samples (from deceased donors and healthy living ND controls) were added to wells of 96-well V-bottom plates (Corning Life Science) and incubated overnight (18 hours) at $37^{\circ} \mathrm{C}$ and $5 \% \mathrm{CO}_{2}$. Plates were then washed with 1\% FBS in PBS and prepared for flow cytometry analysis as described above. Assays were performed using leukocytes from a minimum of 2 healthy living NDs in independent experiments, and mean data were used for calculation of MFI ratios as described above. Data were reported as MFI ratios calculated from the MFIs observed after incubation of leukocytes with the tested DCD, DBD, or ND plasma samples compared with MFIs observed for leukocytes incubated with autologous plasma. 
A
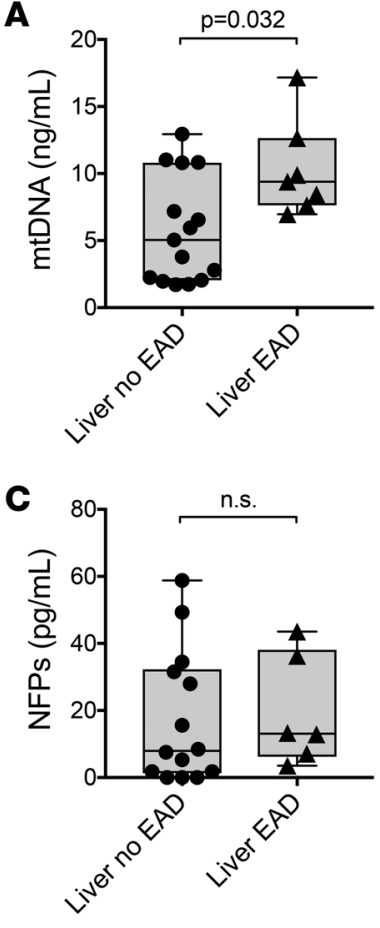

B
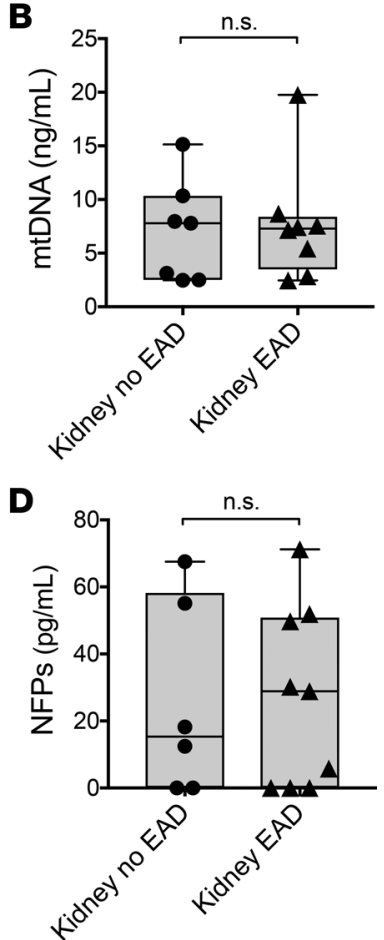

Figure 8. Donor plasma mitochondrial DNA levels correlate with early allograft dysfunction following liver transplantation. (A) Donor plasma mitochondrial DNA (mtDNA) and (C) $\mathrm{N}$-formylated peptide (NFP) levels were compared between liver transplant recipients with or without early allograft dysfunction (EAD), defined as serum AST $>1,500 \mathrm{U} / \mathrm{I}$ within 7 days of transplantation. (B) Donor plasma mtDNA and (D) NFP levels were compared between kidney transplant recipients with or without $E A D$, defined as either slow graft function (SCF, $\mathrm{s} C \mathrm{r} \geq 3$ $\mathrm{mg} / \mathrm{dl}$ at POD5) or delayed graft function (DGF, dialysis requirement within 7 days of transplantation). The gray boxes represent the interquartile range, the lines represent the median, and whiskers indicate the range of observed responses. Comparisons were performed using a Mann-Whitney test. $P<$ 0.05 was considered significant.

Production of ROS by plasma-stimulated PMNs. Red blood cells were lysed from plasma-depleted ACD-preserved blood collected from healthy living NDs as described above. The leukocyte fraction was washed with 1\% FBS PBS, resuspended in serum-free RPMI 1640 medium (Gibco, Thermo Fisher Scientific), and plated at $2.5 \times 10^{5}$ cells per well in one-half area opaque flat-bottom plates (Corning Life Science). Luminol (MilliporeSigma; final concentration $50 \mu \mathrm{g} / \mathrm{ml}$ ) and plasma samples (final dilution 1:2) were added to the wells, and luminescence was recorded every 2 minutes for 10 reads at $37^{\circ} \mathrm{C}$ using a VICTOR Multilabel plate reader (PerkinElmer). fMLP (final concentration $2 \mu \mathrm{M}$ ) was then added to each well, and luminescence was measured every 2 minutes for another 10 reads. Each sample was tested in duplicate, using a minimum of 2 independent experiments performed with leukocytes isolated from different normal healthy donors. Where indicated, mitochondria were depleted from plasma samples by centrifugation at 15,000 $\mathrm{g}$ for 10 minutes, and parallel assays were performed using whole plasma samples and the mitochondria-depleted plasma samples. For inhibition experiments, leukocytes were pretreated with $\mathrm{CsH}$ (Abcam, 1-10 $\mu \mathrm{M}$ ) or diluent control for 5 minutes at room temperature, and $\mathrm{CsH}$ was maintained at the indicated concentration for the duration of the assay. Control experiments were performed using stimulation with PMA $(1 \mu \mathrm{M})$, and LPS $(2 \mu \mathrm{g} / \mathrm{ml})$ was used to confirm that CsH specifically inhibited NFP-induced ROS at the concentrations used.

Serum cytokine and chemokine analysis. Serum cytokine and chemokine concentrations were assayed in duplicate using a 25-plex assay panel (Invitrogen, LHC0009M, Thermo Fisher Scientific) performed according to the manufacturer's recommended protocol and read using a Bio-Plex 200 array reader (BioRad). Data were analyzed using Bio-Plex Manager software (Bio-Rad).

Serum DNA quantification. mtDNA and gDNA were quantified by RT-qPCR. To prevent error associated with DNA purification techniques, we performed RT-qPCR directly on patient sera using a serum-stable DNA polymerase (Omni-KlenTaq-2 DNA Polymerase, DNA Polymerase Technology Inc.) and a PCR enhancer cocktail (PEC-2, DNA Polymerase Technology). Primers specific for COX2 (cytochrome C oxidase subunit II; forward: 5'-ATGACCCACCAATCACATGC-3', reverse: 5'-ATCACATGGCTAGGCCGGAG-3') and GAPDH (forward: 5'-CTGGGAACGAGAGGTTAAGCA-3', reverse: 5'-GGGTCTGGTCACAATATACCACC-3') were used for mtDNA and gDNA, respectively. Final DNA concentrations were determined using standard curves generated with parallel RT-qPCR reactions that used purified mtDNA and gDNA of known concentrations as templates.

Flow cytometry analysis for mitochondria in plasma. Plasma samples were cleared of cellular debris by centrifugation at $1,200 \mathrm{~g}$ for 5 minutes. Plasma microparticles were then pelleted by centrifugation at 
12,000 $\mathrm{g}$ for 15 minutes. The supernatant was removed, and the microparticle pellet was stained with antiTOMM22 antibody (clone EPR13505; catalog ab179826, Abcam) for 10 minutes at room temperature, washed in PBS, and then stained with donkey anti-rabbit IgG-Alexa Fluor 647 polyclonal antibody (catalog ab150075, Abcam). The stained microparticles were rinsed again with PBS and analyzed immediately using a BD LSRII flow cytometer running FACSDiva software (BD Biosciences).

Identification of plasma mitochondria by electron microscopy. Plasma microparticle pellets were prepared as described above. Thin sections were obtained from pelleted plasma microparticles fixed with $3 \%$ glutaraldehyde in $0.1 \mathrm{M}$ cacodylic acid buffer ( $\mathrm{pH}$ 7.4, Ladd Research Industries). The sample was washed 3 times with $0.1 \mathrm{M}$ cacodylic acid buffer and post-stained with $1 \%$ osmium tetroxide in cacodylic buffer for 1 hour. Cells were then washed as before and embedded in $1 \%$ agarose. The agarose containing the cell sample was then prestained with $2 \%$ uranyl acetate (Polaron Instruments Inc.) overnight at $4^{\circ} \mathrm{C}$. The samples were washed and carried through acetone dehydration steps. Infiltration was done using the Epon embedding kit. Samples were sectioned ultrathin $(60-70 \mathrm{~nm})$ on a Reichert Ultracut E ultramicrotome and stained with $2 \%$ uranyl acetate in $50 \%$ ethanol for 30 minutes and SATO's Lead stain for 1 minute. Samples were imaged on a Philips CM12 electron microscope.

Serum NFP quantification. Levels of NFPs present in serum samples were determined by specific immunoassays (Human formylmethionine [fMet] ELISA Kit; MyBioSource Inc.) performed according to the manufacturer's recommended protocol. Samples were tested in duplicate, and absorbance was measured using a VICTOR Multilabel plate reader (PerkinElmer). Concentrations were determined by interpolation of standard curves using Prism 7 software (GraphPad Software Inc.).

Statistics. $P$ values were obtained from Mann-Whitney test (unpaired data) or from Wilcoxon matched-pairs signed-rank test (paired data) for continuous variables and Fisher's exact test for categorical variables. Two-tailed $P$ values of less than 0.05 were considered significant. For multiple comparisons, Kruskal-Wallis with Dunn's multiple comparison test was performed. Spearman's correlation and $P$ values were calculated for fMET, endotoxin, circulating DNA, inflammatory markers, and continuous donor factors, i.e., age, BMI, KDPI, and white blood cell count. $P$ values showed whether the correlation was significantly different from 0 . No adjustment for multiple testing was implemented. Furthermore, each donor characteristic was investigated for its relationship with the biomarkers using Spearman's correlation or Kruskal-Wallis test. No correction for multiple testing was applied. Analyses were performed using GraphPad Prism 7 software or R version 3.4.3.

Study approval. All human samples and data were collected in accordance with protocols approved by the Duke University Institutional Review Board and by Carolina Donor Services (Durham, North Carolina, USA). Signed written informed consent was received from study participants or authorized legal representatives for the use of samples for research purposes prior to inclusion in this study. None of the donors were prisoners at the time of donation. Donor data were obtained from the United Network of Organ Sharing and a research protocol approved by the Carolina Donor Services.

\section{Author contributions}

JP participated in the research design, writing of the paper, conducting experiments, and data analysis. RWE and LL conducted experiments and acquired and analyzed data. VAB participated in data collection. TVB participated in the research design, writing of the paper, and data analysis.

\section{Acknowledgments}

We thank Sara Miller and Ricardo Vancini for their expertise in electron microscopy. We thank Guido Ferrari for comments and suggestions regarding this study. This work was supported by NIH grant AI101263 (to TVB) and a grant from the Duke Department of Surgery (to JP and TVB).

Address correspondence to: Todd Brennan, Cedars-Sinai Comprehensive Transplant Center, 8900 Beverly Boulevard, Los Angeles, California 90048, USA. Phone: 310.423.7408; Email: todd.brennan@cshs.org.

1. United Network for Organ Sharing. https://unos.org/. Accessed July 24, 2018.

2. Amico P. Evolution of graft survival in kidney transplantation: an analysis of the OPTN/UNOS Renal Transplant Registry. Clin Transpl. 2010:1-15.

3. Brennan TV, Lunsford KE, Kuo PC. Innate pathways of immune activation in transplantation. J Transplant. 2010;2010:826240.

4. Cecka JM. The OPTN/UNOS Renal Transplant Registry. Clin Transpl. 2005:1-16. 
5. Stangl M, et al. Influence of brain death on cytokine release in organ donors and renal transplants. Transplant Proc. 2001;33(12):1284-1285.

6. Birks EJ, et al. Elevated tumor necrosis factor-alpha and interleukin-6 in myocardium and serum of malfunctioning donor hearts. Circulation. 2000;102(19 Suppl 3):III352-III358.

7. Murugan R, et al. Increased plasma interleukin- 6 in donors is associated with lower recipient hospital-free survival after cadaveric organ transplantation. Crit Care Med. 2008;36(6):1810-1816.

8. Kono H, Rock KL. How dying cells alert the immune system to danger. Nat Rev Immunol. 2008;8(4):279-289.

9. Rock KL, Latz E, Ontiveros F, Kono H. The sterile inflammatory response. Annu Rev Immunol. 2010;28:321-342.

10. Beg AA. Endogenous ligands of Toll-like receptors: implications for regulating inflammatory and immune responses. Trends Immunol. 2002;23(11):509-512.

11. Johnson GB, Brunn GJ, Platt JL. Activation of mammalian Toll-like receptors by endogenous agonists. Crit Rev Immunol. 2003;23(1-2):15-44.

12. Kono H, Chen CJ, Ontiveros F, Rock KL. Uric acid promotes an acute inflammatory response to sterile cell death in mice. $J$ Clin Invest. 2010;120(6):1939-1949.

13. Bird L. Innate immunity: Linking mitochondria and microbes to inflammasomes. Nat Rev Immunol. 2012;12(4):229.

14. Cloonan SM, Choi AM. Mitochondria: commanders of innate immunity and disease? Curr Opin Immunol. 2012;24(1):32-40.

15. Krysko DV, et al. Emerging role of damage-associated molecular patterns derived from mitochondria in inflammation. Trends Immunol. 2011;32(4):157-164.

16. O'Neill LA. Cardiolipin and the N1rp3 inflammasome. Cell Metab. 2013;18(5):610-612.

17. Shimada K, et al. Oxidized mitochondrial DNA activates the NLRP3 inflammasome during apoptosis. Immunity. 2012;36(3):401-414.

18. West AP, Shadel GS, Ghosh S. Mitochondria in innate immune responses. Nat Rev Immunol. 2011;11(6):389-402.

19. Zhang Q, et al. Circulating mitochondrial DAMPs cause inflammatory responses to injury. Nature. 2010;464(7285):104-107.

20. Zhou R, Yazdi AS, Menu P, Tschopp J. A role for mitochondria in NLRP3 inflammasome activation. Nature. 2011;469(7329):221-225.

21. Emelyanov VV. Mitochondrial connection to the origin of the eukaryotic cell. Eur J Biochem. 2003;270(8):1599-1618.

22. Gray MW, Burger G, Lang BF. Mitochondrial evolution. Science. 1999;283(5407):1476-1481.

23. Hemmi H, et al. A Toll-like receptor recognizes bacterial DNA. Nature. 2000;408(6813):740-745.

24. Le Y, Murphy PM, Wang JM. Formyl-peptide receptors revisited. Trends Immunol. 2002;23(11):541-548.

25. Hauser CJ, Sursal T, Rodriguez EK, Appleton PT, Zhang Q, Itagaki K. Mitochondrial damage associated molecular patterns from femoral reamings activate neutrophils through formyl peptide receptors and P44/42 MAP kinase. J Orthop Trauma. 2010;24(9):534-538.

26. Zhang Q, Itagaki K, Hauser CJ. Mitochondrial DNA is released by shock and activates neutrophils via p38 map kinase. Shock. 2010;34(1):55-59.

27. Sudakov NP, et al. The level of free circulating mitochondrial DNA in blood as predictor of death in case of acute coronary syndrome. Eur J Med Res. 2017;22(1):1

28. Wang L, et al. Plasma nuclear and mitochondrial DNA levels in acute myocardial infarction patients. Coron Artery Dis. 2015;26(4):296-300.

29. Tsai NW, et al. The value of serial plasma nuclear and mitochondrial DNA levels in patients with acute ischemic stroke. Clin Chim Acta. 2011;412(5-6):476-479.

30. Wang HC, et al. Serial plasma deoxyribonucleic acid levels as predictors of outcome in acute traumatic brain injury. J Neurotrauma. 2014;31(11):1039-1045.

31. Garrabou G, et al. The effects of sepsis on mitochondria. J Infect Dis. 2012;205(3):392-400

32. Timmermans K, Kox M, Scheffer GJ, Pickkers P. Plasma nuclear and mitochondrial dna levels, and markers of inflammation, shock, and organ damage in patients with septic shock. Shock. 2016;45(6):607-612.

33. Rao PS, et al. A comprehensive risk quantification score for deceased donor kidneys: the kidney donor risk index. Transplantation. 2009;88(2):231-236.

34. Wenzel-Seifert K, Seifert R. Cyclosporin $\mathrm{H}$ is a potent and selective formyl peptide receptor antagonist. Comparison with N-t-butoxycarbonyl-L-phenylalanyl-L-leucyl-L-phenylalanyl-L- leucyl-L-phenylalanine and cyclosporins A, B, C, D, and E. $J$ Immunol. 1993;150(10):4591-4599.

35. Nanashima A, et al. Analysis of initial poor graft function after orthotopic liver transplantation: experience of an Australian single liver transplantation center. Transplant Proc. 2002;34(4):1231-1235.

36. Brennan TV, Freise CE, Fuller TF, Bostrom A, Tomlanovich SJ, Feng S. Early graft function after living donor kidney transplantation predicts rejection but not outcomes. Am J Transplant. 2004;4(6):971-979.

37. Irish WD, Ilsley JN, Schnitzler MA, Feng S, Brennan DC. A risk prediction model for delayed graft function in the current era of deceased donor renal transplantation. Am J Transplant. 2010;10(10):2279-2286.

38. Pratschke J, et al. Accelerated rejection of renal allografts from brain-dead donors. Ann Surg. 2000;232(2):263-271.

39. Yarlagadda SG, et al. Marked variation in the definition and diagnosis of delayed graft function: a systematic review. Nephrol Dial Transplant. 2008;23(9):2995-3003.

40. Barklin A. Systemic inflammation in the brain-dead organ donor. Acta Anaesthesiol Scand. 2009;53(4):425-435.

41. Lopau K, Mark J, Schramm L, Heidbreder E, Wanner C. Hormonal changes in brain death and immune activation in the donor. Transpl Int. 2000;13 Suppl 1:S282-S285.

42. Pratschke J, Wilhelm MJ, Kusaka M, Hancock WW, Tilney NL. Activation of proinflammatory genes in somatic organs as a consequence of brain death. Transplant Proc. 1999;31(1-2):1003-1005.

43. van Der Hoeven JA, et al. Effects of brain death and hemodynamic status on function and immunologic activation of the poten tial donor liver in the rat. Ann Surg. 2000;232(6):804-813

44. Lord JM, et al. The systemic immune response to trauma: an overview of pathophysiology and treatment. Lancet. 2014;384(9952):1455-1465. 
45. Shi Y, Evans JE, Rock KL. Molecular identification of a danger signal that alerts the immune system to dying cells. Nature. 2003;425(6957):516-521.

46. Maeda A, Fadeel B. Mitochondria released by cells undergoing TNF- $\alpha$-induced necroptosis act as danger signals. Cell Death Dis 2014;5:e1312

47. McDonald B, et al. Intravascular danger signals guide neutrophils to sites of sterile inflammation. Science. 2010;330(6002):362-366.

48. Arnoult D, Soares F, Tattoli I, Girardin SE. Mitochondria in innate immunity. EMBO Rep. 2011;12(9):901-910.

49. Ries M, Schuster P, Thomann S, Donhauser N, Vollmer J, Schmidt B. Identification of novel oligonucleotides from mitochondrial DNA that spontaneously induce plasmacytoid dendritic cell activation. J Leukoc Biol. 2013;94(1):123-135.

50. Hazeldine J, Hampson P, Opoku FA, Foster M, Lord JM. N-Formyl peptides drive mitochondrial damage associated molecular pattern induced neutrophil activation through ERK1/2 and P38 MAP kinase signalling pathways. Injury. 2015;46(6):975-984

51. Suresh MV, et al. Toll-Like Receptor-9 (TLR9) is requisite for acute inflammatory response and injury following lung contusion. Shock. 2016;46(4):412-419.

52. Calfee CS, Matthay MA. Clinical immunology: Culprits with evolutionary ties. Nature. 2010;464(7285):41-42.

53. Zimmer C. Origins. On the origin of eukaryotes. Science. 2009;325(5941):666-668.

54. Miller LS, et al. Inflammasome-mediated production of IL-1beta is required for neutrophil recruitment against Staphylococcus aureus in vivo. J Immunol. 2007;179(10):6933-6942.

55. Weber DJ, et al. The HMGB1-RAGE axis mediates traumatic brain injury-induced pulmonary dysfunction in lung transplantation. Sci Transl Med. 2014;6(252):252ra124.

56. Gu X, Yao Y, Wu G, Lv T, Luo L, Song Y. The plasma mitochondrial DNA is an independent predictor for post-traumatic systemic inflammatory response syndrome. PLoS ONE. 2013;8(8):e72834.

57. Hernández-Jiménez E, et al. Circulating monocytes exhibit an endotoxin tolerance status after acute ischemic stroke: Mitochondrial dna as a putative explanation for poststroke infections. J Immunol. 2017;198(5):2038-2046.

58. Yamanouchi S, Kudo D, Yamada M, Miyagawa N, Furukawa H, Kushimoto S. Plasma mitochondrial DNA levels in patients with trauma and severe sepsis: time course and the association with clinical status. J Crit Care. 2013;28(6):1027-1031.

59. Zhao C, Itagaki K, Gupta A, Odom S, Sandler N, Hauser CJ. Mitochondrial damage-associated molecular patterns released by abdominal trauma suppress pulmonary immune responses. J Trauma Acute Care Surg. 2014;76(5):1222-1227.

60. Simmons JD, et al. Elevated levels of plasma mitochondrial DNA DAMPs are linked to clinical outcome in severely injured human subjects. Ann Surg. 2013;258(4):591-598.

61. Perfetto SP, Ambrozak D, Nguyen R, Chattopadhyay PK, Roederer M. Quality assurance for polychromatic flow cytometry using a suite of calibration beads. Nat Protoc. 2012;7(12):2067-2079. 